\title{
Some Discrete Competition Models and the Competitive Exclusion Principle ${ }^{\dagger}$
}

\author{
J.M. CUSHING ${ }^{a, *}$, SHEREE LEVARGE ${ }^{a}$, NAKUL CHITNIS ${ }^{a}$ and \\ SHANDELLE M. HENSON ${ }^{\mathrm{b}}$ \\ a Department of Mathematics, Program on Applied Mathematics, University of Arizona, \\ Tucson, AZ 85721, USA; 'bepartment of Mathematics, Andrews University, Berrien Springs, \\ MI 49104, USA
}

(Received 6 May 2003; In final form 11 November 2003)

Dedicated to Professor Saber Elaydi on the Occasion of His 60th Birthday

A difference equation model, called that Leslie/Gower model, played a key historical role in laboratory experiments that helped establish the "competitive exclusion principle" in ecology. We show that this model has the same dynamic scenarios as the famous Lotka/Volterra (differential equation) competition model. It is less well known that some anomalous results from the experiments seem to contradict the exclusion principle and Lotka/Volterra dynamics. We give an example of a competition model that has non-Lotka/Volterra dynamics that are consistent with the anomalous case.

Keywords: Difference equations; Global stability; Competition models; Competitive exclusion principle; Leslie/Gower model; Lotka/Volterra dynamics

AMS Nos: 39A11; 92D40

\section{INTRODUCTION}

A fundamental tenet in theoretical ecology is the "competitive exclusion principle". According to this tenet, two similar species competing for a limited resource cannot coexist; one of the species will be driven to extinction. This principle is supported by many mathematical models, the most famous of which is the Lotka/Volterra differential equation model for two competing species. It is well known that the Lotka/Volterra model allows just four dynamic scenarios, all of which involve only equilibria as possible asymptotic states. A coexistence case (in the form of a globally asymptotically stable equilibrium) occurs if the competition between the species is weak. If, however, the inter-species competition is sufficiently strong, then competitive exclusion occurs (in the form of an equilibrium state possessing one zero component). The competitive exclusion case has three possible dynamic scenarios, depending upon relationships among the model coefficients. Two of these scenarios are symmetric cases that have globally attracting equilibria in which one species is absent. The third, and final scenario, has an unstable (saddle) coexistence

\footnotetext{
${ }^{\dagger}$ This research was supported by National Science Foundation grant DMS 9973126.

*Corresponding author. E-mail: cushing@math.arizona.edu 
equilibrium whose stable manifold determines two basins of attraction, one for each of the competitive exclusion equilibria. In this "saddle" case, the species that goes extinct is determined by the initial conditions of both species.

During the 1940s, 50s and 60s, laboratory experiments played a key role in establishing the competitive exclusion principle in theoretical ecology. One series of laboratory studies, which today is still cited in text books, was conducted by Park and Mertz using two species of flour beetles (of the genus Tribolium) [37-40]. Park and his collaborators, Leslie and Gower, used a difference equation model in these studies, rather than the Lotka/Volterra differential equations [26]. Although they did not give a mathematical analysis of their model, they worked under the assumption that it possesses the same dynamic scenarios as the Lotka/Volterra model.

It is interesting that although Park's experiments were considered a validation of the competitive exclusion principle, there were some anomalous results. In one experiment competitive exclusion did not always occur. Whether competitive coexistence occurred or competitive exclusion occurred (and how) depended on the initial population numbers given the two species. This result puzzled Park and his collaborators, since this dynamic scenario is not allowed by Lotka/Volterra-type competition models which guided their thinking [14,27]. A natural question to examine, from a mathematical point of view, is whether or not there exist any two species competition models that allow for such a multiple attractor, coexistence/exclusion case. A number of studies involving competition models have, under certain circumstances, found results of non-Lotka/Volterra type or that contradicted the competitive exclusion principle in one way or another $[2,5-8,10,13,20,22-24,28,29,31-36,41,42]$. None of these results, however, involve the multiple attractor scenario described above.

The Leslie/Gower competition model was studied by Liu and Elaydi, who showed that all bounded orbits converge to an equilibrium in an eventually monotonic manner (using the theory of discrete monotone flows) [30]. In this paper we will extend this result by showing that the Leslie/Gower model has the same dynamic scenarios as the Lotka/Volterra model (characterizing these cases according to model parameters). We will also investigate a competition model that does conform not to the Lotka/Volterra cases and that allows for multiple, coexistence and exclusion, attractors.

\section{THE LESLIE/GOWER MODEL}

If $b>1$ all solutions of the Beverton/Holt equation

$$
x_{t+1}=b \frac{1}{1+c_{11} x_{t}} x_{t}
$$

with $x_{0}>0$ tend (monotonically) to the equilibrium $x=(b-1) / c_{11}$. This difference equation is an appropriate analog of the logistic differential equation [12]. Just as the famous Lotka/Volterra two species (differential equation) competition model is a modification of the logistic differential equation, the Leslie/Gower (difference equation) competition model [26]

$$
\begin{aligned}
& x_{t+1}=b_{1} \frac{1}{1+c_{11} x_{t}+c_{12} y_{t}} x_{t} \\
& y_{t+1}=b_{2} \frac{1}{1+c_{21} x_{t}+c_{22} y_{t}} y_{t}
\end{aligned}
$$

is a modification of the Beverton/Holt equation. In this model all coefficients are positive and we can therefore scale $x$ and $y$ so that $c_{11}=c_{22}=1$. Without loss in generality we consider 
the system

$$
\begin{aligned}
& x_{t+1}=b_{1} \frac{1}{1+x_{t}+c_{1} y_{t}} x_{t} \\
& y_{t+1}=b_{2} \frac{1}{1+c_{2} x_{t}+y_{t}} y_{t}
\end{aligned}
$$

where $b_{i}>0$ and $c_{i}>0$. We denote solutions of this system by $\left(x_{t}, y_{t}\right), t=0,1,2,3, \ldots$ (For some results concerning difference equations defined by rational functions see Refs. [3,4,25]. The results in these papers do not apply to the Leslie/Gower model, however. Other papers that deal with discrete competition models include [16-19].)

In population dynamic applications we are interested in solutions with non-negative components $x_{t} \geq 0, y_{t} \geq 0$. Let $R_{+}^{2} \stackrel{\circ}{=}[0,+\infty) \times[0,+\infty)$ and $\stackrel{\circ}{R}_{+}^{2} \triangleq(0,+\infty) \times(0,+\infty)$

$$
\phi:(x, y) \rightarrow\left(b_{1} \frac{1}{1+x+c_{1} y} x, \quad b_{2} \frac{1}{1+c_{2} x+y} y\right)
$$

takes $R_{+}^{2}$ into itself. The same is true of $\stackrel{\circ}{R}_{+}^{2}$ and of the coordinate axes $[0,+\infty) \times\{0\}$ and $\{0\} \times[0,+\infty)$. Moreover, all solutions in $R_{+}^{2}$ are forward bounded. Specifically, $\phi: R_{+}^{2} \rightarrow S \stackrel{\circ}{=}\left[0, b_{1}\right) \times\left[0, b_{2}\right)$. It follows from Proposition 1 in Ref. [30] that all orbits in $R_{+}^{2}$ approach an equilibrium as $t \rightarrow+\infty$.

The map $\phi$ is also invertible on $R_{+}^{2}$, since for $\left(x^{\prime}, y^{\prime}\right) \in S$ in the range of $\phi$ the equations

$$
b_{1} \frac{1}{1+x+c_{1} y} x=x^{\prime}, \quad b_{2} \frac{1}{1+c_{2} x+y} y=y^{\prime}
$$

have the unique solution

$$
x=\frac{\beta_{2}-1+c_{1}}{\Delta}, \quad y=\frac{\beta_{1}-1+c_{2}}{\Delta}
$$

where

$$
\beta_{1} \stackrel{\circ}{=} \frac{b_{1}}{x^{\prime}}>1, \quad \beta_{2} \stackrel{\circ}{=} \frac{b_{2}}{y^{\prime}}>1, \quad \Delta \stackrel{\circ}{=}\left(\beta_{1}-1\right)\left(\beta_{2}-1\right)-c_{1} c_{2}
$$

(the range of $\phi$ is defined by the inequality $\Delta>0$ ). The formulas for the pre-images $x$ and $y$ show the inverse $\phi^{-1}$ continuous.

Lemma 1 The map $\phi: R_{+}^{2} \rightarrow S$ is one-one and bicontinuous.

The points $E_{0}:(0,0) ; E_{1}:\left(b_{1}-1,0\right) ; E_{2}:\left(0, b_{2}-1\right)$ are fixed points of the map $\phi$ (i.e. are equilibria of the Leslie/Gower model (1) and (2)). These are "exclusion" equilibria.

The set of points whose $x$-coordinate is held fixed by the map $\phi$ is the line $x+c_{1} y=$ $b_{1}-1$. If this line intersects $\stackrel{\circ}{+}_{+}^{2}$ (i.e. if $b_{1}>1$ ), we denote the resulting line segment by $L_{1}$. Similarly, if $b_{2}>1$, the points on the line segment $L_{2}$ from the line $c_{2} x+y=b_{2}-1$ lying in $R_{+}^{2}$ is the set of points in $R_{+}^{2}$ whose $y$-coordinate is held fixed by the map $\phi$. If $b_{1}>1$ the map $\phi$ takes a point $(x, y) \in R_{+}^{2}$ lying above (below) $L_{1}$ to a point with smaller (larger) $x$-coordinate. If $b_{2}>1$ the map $\phi$ takes a point $(x, y) \in R_{+}^{2}$ lying above (below) $L_{2}$ to a point with smaller (larger) $y$-coordinate.

The only other fixed point of $\phi$ is

$$
E_{3}:\left(\frac{b_{2}-1}{c_{1} c_{2}-1}\left(c_{1}-\frac{b_{1}-1}{b_{2}-1}\right), \quad \frac{b_{1}-1}{c_{1} c_{2}-1}\left(c_{2}-\frac{b_{2}-1}{b_{1}-1}\right)\right)
$$


This equilibrium lies in $R_{+}^{2}$ if and only if $b_{1}>1, b_{2}>1$ and $L_{1}$ and $L_{2}$ intersect in $R_{+}^{2}$. This is a "coexistence" equilibrium.

An equilibrium is globally asymptotically stable on $R_{+}^{2}\left(\right.$ or $\stackrel{\circ}{+}_{+}^{2}$ ) if it is locally asymptotically stable (on $R^{2}$ ) and if $\left(x_{0}, y_{0}\right) \in R_{+}^{2}$ (or $\stackrel{\circ}{+}_{+}^{2}$ ) implies that $\left(x_{t}, y_{t}\right)$ tends to the equilibrium as $t \rightarrow+\infty$.

The Jacobian of Eqs. (1) and (2) is

$$
J=\left(\begin{array}{cc}
-b_{1} \frac{1}{\left(1+x+c_{1} y\right)^{2}} x+b_{1} \frac{1}{1+x+c_{1} y} & -b_{1} c_{1} \frac{1}{\left(1+x+c_{1} y\right)^{2}} x \\
-b_{2} c_{2} \frac{1}{\left(1+c_{2} x+y\right)^{2}} y & -b_{2} \frac{1}{\left(1+c_{2} x+y\right)^{2}} y+b_{2} \frac{1}{1+c_{2} x+y}
\end{array}\right)
$$

The Jacobians evaluated at $E_{0}, E_{1}$ and $E_{2}$ are

$$
J_{0}=\left(\begin{array}{cc}
b_{1} & 0 \\
0 & b_{2}
\end{array}\right), \quad J_{1}=\left(\begin{array}{cc}
\frac{1}{b_{1}} & \frac{1}{b_{1}}\left(1-b_{1}\right) c_{1} \\
0 & \frac{b_{2}}{1+\left(b_{1}-1\right) c_{2}}
\end{array}\right), \quad J_{2}=\left(\begin{array}{cc}
\frac{b_{1}}{1+\left(b_{2}-1\right) c_{1}} & 0 \\
\frac{1}{b_{2}}\left(1-b_{2}\right) c_{2} & \frac{1}{b_{2}}
\end{array}\right)
$$

respectively. Their eigenvalues appear along the diagonals.

LEMMA 2

(a) If $b_{1}<1, b_{2}<1$ then $E_{0}$ is globally asymptotically stable on $R_{+}^{2}$.

(b) If $b_{1}>1, b_{2}<1$ then $E_{1}$ is globally asymptotically stable on $\stackrel{\circ}{+}_{+}^{2}$.

(c) If $b_{1}<1, b_{2}>1$ then $E_{2}$ is globally asymptotically stable on $\stackrel{\circ}{+}_{+}^{2}$.

Proof

(a) The eigenvalues of $J_{0}$ are less than 1 and $E_{0}$ is locally asymptotically stable. Since $E_{0}$ is the only equilibrium in $R_{+}^{2}$ it follows from the Liu and Elaydi's theorem that all solutions in $R_{+}^{2}$ converge to $E_{0}$. This can also be seen, more directly, from the inequalities

$$
\begin{aligned}
& 0 \leq x_{t+1}<b_{1} x_{t} \\
& 0 \leq y_{t+1}<b_{2} y_{t}
\end{aligned}
$$

and an induction argument.

(b) The only equilibria in $R_{+}^{2}$ are $E_{0}$ and $E_{1}$. From $J_{0}$ we see that $E_{0}$ is a saddle. Since the coordinate axes are invariant, the stable manifold is the $y$-axis (since by Eq. (4) $y_{t} \rightarrow 0$ ). From the stable manifold and Hartman/Grobman theorems for maps [15,21], it follows that no solution in $\stackrel{\circ}{+}_{+}^{2}$ can approach $E_{0}$. By Liu and Elaydi's theorem all solutions must therefore approach $E_{1}$. The eigenvalues of $J_{1}$ are less than 1 and $E_{1}$ is locally asymptotically stable.

(c) This symmetric case is proved in manner analogous to case (b).

From now on we assume $b_{1}>1, b_{2}>1$. These inequalities imply $E_{1}$ and $E_{2}$ lie on the positive $x$ - and $y$-axis, respectively. We distinguish four cases depending on the orientation of the line segments $L_{1}$ and $L_{2}$ as shown in Figure 1. The inequalities satisfied by the coefficients that correspond to these cases can be easily read from the relative positions of the intercepts of $L_{1}$ and $L_{2}$ in Figure 1. In particular, the following inequalities characterize 

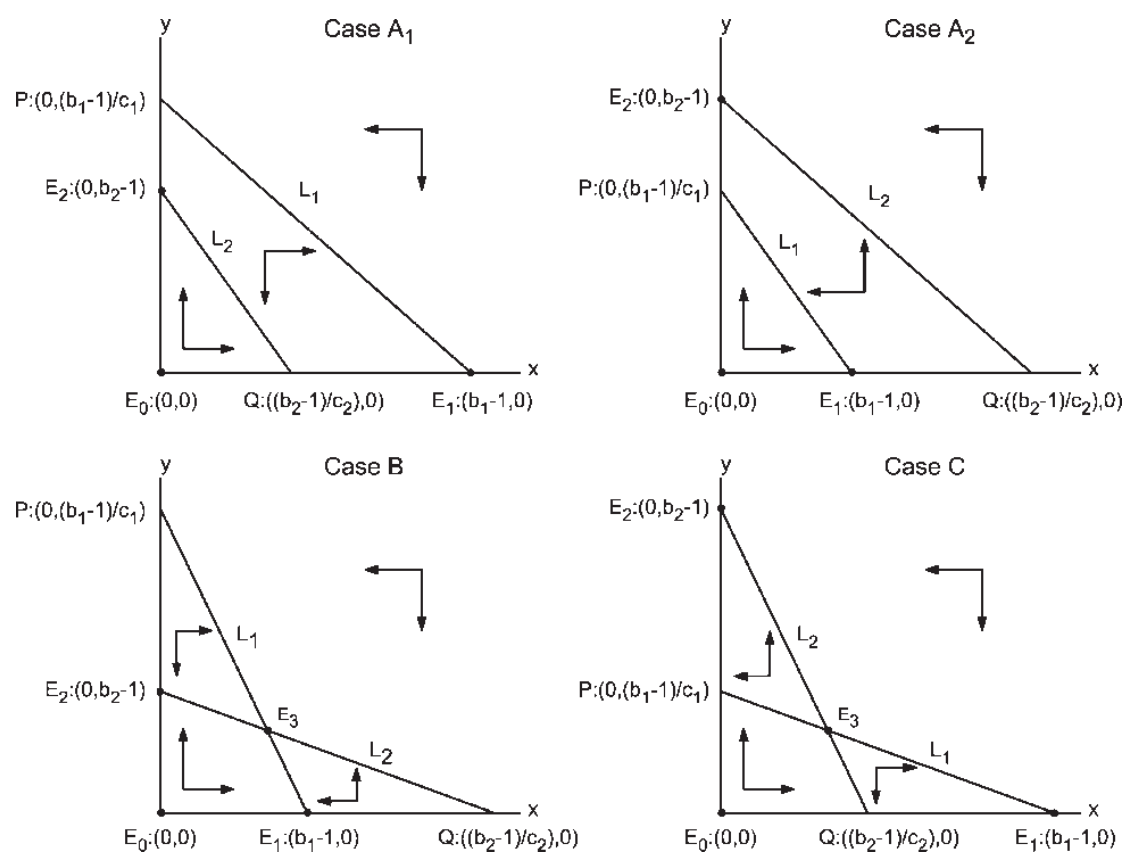

FIGURE 1 The four possible orientations of $L_{1}$ and $L_{2}$.

Cases B and C:

$$
\begin{array}{lll}
\text { Case B }: & c_{1}\left(b_{2}-1\right)<b_{1}-1, \quad c_{2}\left(b_{1}-1\right)<b_{2}-1 \\
\text { Case C }: & c_{1}\left(b_{2}-1\right)>b_{1}-1, & c_{2}\left(b_{1}-1\right)>b_{2}-1 .
\end{array}
$$

From these inequalities we find that

$$
\begin{aligned}
& c_{1} c_{2}<1 \text { holds in Case B } \\
& c_{1} c_{2}>1 \text { holds in Case C. }
\end{aligned}
$$

Lemma 3 Assume $b_{1}>1$ and $b_{2}>1$. The equilibrium $E_{0}$ is a repellor. For $i=1,2$ the equilibrium $E_{i}$ is locally asymptotically stable in Cases $A_{i}$ and $C$, but is a saddle in Cases $A_{j}$ $(j \neq i)$ and $B$. In the latter two cases, the stable manifold of the saddle $E_{i}$ is the positive coordinate axis on which it lies. Equilibrium $E_{3}$ is locally asymptotically stable in Case B and is a saddle in Case C.

Proof The eigenvalues of $J_{0}$ are $b_{1}>1, b_{2}>1$ and $E_{0}$ is therefore a repellor. The eigenvalue $1 / b_{1}$ of $J_{1}$ is less than one. Solutions starting on the positive $x$-axis satisfy the Bervton/Holt equation (with $b_{1}>1$ ) and therefore approach $E_{1}$. The second eigenvalue

$$
\frac{b_{2}}{1+c_{2}\left(b_{1}-1\right)}
$$

is less than one in Cases $A_{1}$ and $C$ and greater than one in Cases $A_{2}$ and $B$. A similar calculation shows the result for $E_{2}$. 
Finally, we consider the equilibrium $E_{3}$, at which the Jacobian is

$$
J_{3}=\left(\begin{array}{cc}
\frac{c_{1} c_{2} b_{1}-c_{1} b_{2}+c_{1}-1}{b_{1}\left(c_{1} c_{2}-1\right)} & c_{1} \frac{b_{1}-c_{1} b_{2}+c_{1}-1}{b_{1}\left(c_{1} c_{2}-1\right)} \\
c_{2} \frac{-c_{2} b_{1}+b_{2}+c_{2}-1}{b_{2}\left(c_{1} c_{2}-1\right)} & \frac{-c_{2} b_{1}+c_{2} c_{1} b_{2}+c_{2}-1}{b_{2}\left(c_{1} c_{2}-1\right)}
\end{array}\right)
$$

This equilibrium is locally asymptotically stable if the Jury conditions

$$
\left|\operatorname{tr} J_{3}\right|<1+\operatorname{det} J_{3}<2
$$

hold, where $\operatorname{det} J_{3}$ is the determinant and $\operatorname{tr} J_{3}$ is the trace of $J_{3}$ [1]. If at least one of these inequalities is reversed, then the equilibrium is unstable. The inequalities are equivalent to the following three inequalities

(a) $1+\operatorname{det} J_{3}<2$

(b) $-1-\operatorname{det} J_{3}<\operatorname{tr} J_{3}$

(c) $\operatorname{tr} J_{3}<1+\operatorname{det} J_{3}$.

A calculation shows

$$
\begin{aligned}
\operatorname{det} J_{3} & =\frac{c_{2}\left(c_{1}-1\right)\left(b_{1}-1\right)+c_{1}\left(c_{2}-1\right)\left(b_{2}-1\right)+c_{1} c_{2}-1}{b_{1} b_{2}\left(c_{1} c_{2}-1\right)} \\
\operatorname{tr} J_{3} & =\frac{2 b_{2} b_{1} c_{1} c_{2}-b_{2}+c_{1} b_{2}-c_{1} b_{2}^{2}-b_{1}+c_{2} b_{1}-c_{2} b_{1}^{2}}{b_{1} b_{2}\left(c_{1} c_{2}-1\right)} .
\end{aligned}
$$

Using these formulas, we find that inequality (a) is equivalent to

$$
\left(b_{1}-1\right)\left(b_{2}-1\right)+\frac{c_{1}\left(b_{2}-1\right)-\left(b_{1}-1\right)}{c_{1} c_{2}-1}+\frac{c_{2}\left(b_{1}-1\right)-\left(b_{2}-1\right)}{c_{1} c_{2}-1}>0 .
$$

By Eqs. (5) and (6) this inequality holds. Inequality (b) is equivalent to

$$
\left(b_{1}+1\right)\left(b_{2}+1\right)+c_{2}\left(b_{1}+1\right) \frac{c_{1}\left(b_{2}-1\right)-\left(b_{1}-1\right)}{c_{1} c_{2}-1}+c_{1}\left(b_{2}+1\right) \frac{c_{2}\left(b_{1}-1\right)-\left(b_{2}-1\right)}{c_{1} c_{2}-1}>0
$$

which Eqs. (5) and (6) show also holds true. Finally, we consider inequality (c), which it turns out is equivalent to

$$
\frac{\left(c_{1}\left(b_{2}-1\right)-\left(b_{1}-1\right)\right)\left(c_{2}\left(b_{1}-1\right)-\left(b_{2}-1\right)\right)}{c_{1} c_{2}-1}<0 .
$$

From Eqs. (5) and (6), we see that this inequality holds in Case B and, as a result, $E_{3}$ is locally asymptotically stable. In Case $\mathrm{C}$, however, the reverse inequality holds and $E_{3}$ is unstable.

To see, in the latter case, that $E_{3}$ is in fact a saddle we note that the characteristic quadratic polynomial of $J_{3}$, namely,

$$
p(\lambda)=\lambda^{2}-\left(\operatorname{tr} J_{3}\right) \lambda+\operatorname{det} J_{3}
$$


satisfies $p(1)=1-\operatorname{tr} J_{3}+\operatorname{det} J_{3}<0$ (because the inequality in (c) is reversed in Case C), $p(-1)=1+\operatorname{tr} J_{3}+\operatorname{det} J_{3}>0$ (because (b) holds), and $p(+\infty)=+\infty$. Thus, $p(\lambda)$ has a positive root $\lambda>1$ and a root between -1 and +1 .

THEOREM 4 Consider the Leslie/Gower competition model (1) and (2) with $b_{i}>1$. Each component $x_{t}$ and $y_{t}$ of a solution in $\stackrel{\circ}{R}_{+}^{2}$ is eventually monotonic.

(a) In Case $A_{i}$, the equilibrium $E_{i}$ is globally asymptotically stable on $\stackrel{\circ}{R}_{+}^{2}$.

(b) In Case $B$, the equilibrium $E_{3}$ is globally asymptotically stable on $\stackrel{\circ}{R}_{+}^{2}$.

(c) In Case $C$, for solution in $\stackrel{\circ}{R}_{+}^{2}$ tends to one of the locally asymptotically stable equilibria $E_{1}$ or $E_{2}$ or to the saddle equilibrium $E_{3}$ as $t \rightarrow+\infty$.

Proof Every solution in $\stackrel{\circ}{R}_{+}^{2}$ is eventually monotonic and converges to one of the four equilibria $E_{i}$ by Liu and Erlaydi's theorem.

(a) The two Cases $A_{1}$ and $A_{2}$ are symmetric and we consider Case $A_{1}$ only. Observe that the (closed) triangular region $E_{0} E_{1} P$ is forward invariant under the map $\phi$. This follows from two facts: $\phi$ is one-to-one and bicontinuous (Lemma 1) and the boundary of $E_{0} E_{1} P$ maps into $E_{0} E_{1} P$ (see Fig. 1). Thus, if a solution starts in the triangle $E_{0} E_{1} P$ is remains there and as a result the component $x_{t}$ is increasing. It follows that the solution approaches $E_{1}$. If, on the other hand, $\left(x_{0}, y_{0}\right) \in \stackrel{\circ}{R}_{+}^{2}$ lies outside the triangle $E_{0} E_{1} P$ there are two options: either the solution sequence $\left(x_{t}, y_{t}\right)$ enters the triangle in a finite number of steps, in which case it will remain there and converge to $E_{1}$ (as we just proved), or it remains outside the triangle for all $t$. In the latter case, the solution can only converge to $E_{1}$. In summary, $\left(x_{0}, y_{0}\right) \in \stackrel{\circ}{R}_{+}^{2}$ implies $\left(x_{t}, y_{t}\right)$ tends $E_{1}$ as $t \rightarrow+\infty$. This, together with Lemma 3 , shows $E_{1}$ is globally asymptotically stable on $\stackrel{\circ}{R}_{+}^{2}$.

(b) By Lemma $3, E_{3}$ is locally asymptotically stable. The discrete stable manifold and Hartman/Grobman theorems, together with Lemma 3, imply that no orbit in $\stackrel{\circ}{+}_{+}^{2}$ can approach $E_{0}, E_{1}$ or $E_{2}$. It follows from Liu and Elaydi's theorem that all orbits in $\stackrel{\circ}{R}_{+}^{2}$ approach $E_{3}$.

(c) By Lemma $3, E_{0}$ is a repellor and cannot be approached by a solution in $\stackrel{\circ}{+}_{+}^{2}$.

For case (c) in Theorem 4 we do not have a complete characterization of the basins of attraction for each equilibrium. From the stable manifold theorem for maps [15,21] we know the local stable manifold of $E_{3}$ is one dimensional (since by Lemma 2 the equilibrium $E_{3}$ is a hyperbolic saddle in Case $\mathrm{C}$ ), but we do not know if this is globally true in $R_{+}^{2}$. We can show that the basin of attraction of $E_{1}$ contains the interior of the closed triangle $Q E_{1} E_{3}$. To see this notice that $\phi$ maps the boundary of the triangle into the triangle: $\phi: \partial\left(Q E_{1} E_{3}\right) \rightarrow Q E_{1} E_{3}$ (see Fig. 1). Since $\phi$ is one-one and bicontinuous, it follows that $\phi: Q E_{1} E_{3} \rightarrow Q E_{1} E_{3}$ (i.e. $Q E_{1} E_{3}$ is forward invariant). Solutions starting in (or eventually entering) the interior of $Q E_{1} E_{3}$ must accordingly approach the equilibrium $E_{1}$. Similarly, one can show that the basin of attraction of $E_{2}$ contains the interior of the triangle $P E_{2} E_{3}$.

The four dynamic cases in Theorem 4 (and Fig. 1) are the same as those in the Lotka/Volterra model and have the same ecological interpretation. Coexistence (Case B) occurs if interspecific competition is weak in the sense that the coefficients $c_{i}$ are small:

$$
c_{1}<\frac{b_{1}-1}{b_{2}-1}, \quad c_{2}<\frac{b_{2}-1}{b_{1}-1} .
$$


If interspecific competition is too large (one or both inequalities are reversed), then competitive exclusion occurs. This is the theoretical foundation for the classical competitive exclusion principle. It is because of this principle that the anomolous case observed in some of Park's experiments were puzzling. In the next section, we will study a competition model which does not support this exclusion principle- a model which in fact predicts (nonequilibrium) coexistence when the competition coefficients are increased.

\section{A JUVENILE/ADULT RICKER MODEL}

Since the Leslie/Gower competition model (1) and (2) has only the Lotka/Volterra dynamics described in Theorem 4, this model offers no explanation of the multiple attractor case (with initial condition dependent coexistence or exclusion) observed by Park. A discrete competition model that does provide an explanation is the so-called LPA model [10-12]. This six dimensional model is based on three life cycle stages for each species and was derived explicitly to study the dynamics of flour beetle species. See Ref. [14] for an account of the LPA competition model and some of its non-Lotka/Volterra dynamics that contradict the classical competitive exclusion principle.

A mathematical exploration of competition models that possess multiple attractor cases with initial condition dependent coexistence or exclusion might start with the Leslie/Gower model and try to determine what kinds of structural changes in the model will result in non-Lotka/Volterra dynamics and contradictions to the competitive exclusion principle. For example, if exponential non-linearities replace the rational function non-linearities of the Leslie/Gower model, one obtains the Ricker competition model

$$
\begin{aligned}
& x_{t+1}=b_{1} x_{t} \exp \left(-c_{11} x_{t}-c_{12} y_{t}\right) \\
& y_{t+1}=b_{2} y_{t} \exp \left(-c_{21} x_{t}-c_{22} y_{t}\right)
\end{aligned}
$$

(exponential non-linearities also appear in the LPA model). Numerical investigations of this model have uncovered certain kinds of non-Lotka/Volterra dynamics [10], but not a case of multiple attractors with both coexistence and exclusion. Whether or not such multiple attractor cases can occur in this system remains an interesting open question.

From a mathematical point of view life cycle stages (such as appear in the LPA model) introduce time delays. A modification of the Ricker competition model, in which individuals from one of the two species are characterized by their reproductive maturity, is described by the difference equations

$$
\begin{aligned}
& J_{t+1}=b_{1} A_{t} \exp \left(-c_{11} A_{t}-c_{12} y_{t}\right) \\
& A_{t+1}=(1-\mu) J_{t} \\
& y_{t+1}=b_{2} y_{t} \exp \left(-c_{21} J_{t}-c_{22} y_{t}\right) .
\end{aligned}
$$

Here $J_{t}$ and $A_{t}$ are the numbers of juveniles and adults at time $t$ of the species $x$. Species $y$ in this model remains unstructured. The parameter $\mu(0 \leq \mu<1)$ is the juvenile mortality rate.

In this paper, we will not attempt a thorough analysis of the juvenile/adult Ricker competition model (7). We will only show that under certain conditions the model possesses three attractors, two exclusion (equilibrium) attractors and one (non-equilibrium) coexistence attractor. 
The exclusion equilibria for Eq. (7) are

$$
\begin{aligned}
& E_{0}:(J, A, y)=(0,0,0) \\
& E_{1}:(J, A, y)=\left(\frac{\ln n}{c_{11}(1-\mu)}, \frac{\ln n}{c_{11}}, 0\right) \\
& E_{2}:(J, A, y)=\left(0,0, \frac{\ln b_{2}}{c_{22}}\right) .
\end{aligned}
$$

Here we have defined $n \stackrel{\circ}{=} b_{1}(1-\mu)$ [9]. We assume $n>1, b_{2}>1$ so that these equilibria are non-negative. The Jacobian at $E_{0}$

$$
\left(\begin{array}{ccc}
0 & b_{1} & 0 \\
-\mu+1 & 0 & 0 \\
0 & 0 & b_{2}
\end{array}\right)
$$

has eigenvalues $\lambda=b_{2}$ and $\pm \sqrt{n}$. The Jacobian at $E_{1}$

$$
\left(\begin{array}{ccc}
0 & \frac{1-\ln n}{n} b_{1} & -\frac{b_{1} c_{12} \ln n}{c_{21} n} \\
1-\mu & 0 & 0 \\
0 & 0 & b_{2} \exp \left(-\frac{c_{21} \ln n}{c_{11}(1-\mu)}\right)
\end{array}\right)
$$

has eigenvalues $\lambda= \pm \sqrt{1-\ln n}$ and $b_{2} \exp \left(-c_{21} \ln n / c_{11}(1-\mu)\right)$. The Jacobian at $E_{2}$

$$
\left(\begin{array}{ccc}
0 & b_{1} e^{-\frac{c_{12}}{c_{22}} \ln b_{2}} & 0 \\
1-\mu & 0 & 0 \\
-\frac{c_{21}}{c_{22}} \ln b_{2} & 0 & 1-\ln b_{2}
\end{array}\right)
$$

has eigenvalues $\lambda= \pm \sqrt{n}$ and $1-\ln b_{2}$.

Theorem 5 Assume $n=b_{1}(1-\mu)>1, b_{2}>1$ in the juvenile/adult Ricker competition model (7).

(a) $E_{0}$ is a repellor.

(b) $E_{1}$ is locally asymptotically stable if

$$
1<n<e^{2}, \quad c_{11}(1-\mu) \frac{\ln b_{2}}{\ln n}<c_{21} .
$$

(c) $E_{2}$ is locally asymptotically stable if

$$
1<b_{2}<e^{2}, \quad c_{22} \frac{\ln n}{\ln b_{2}}<c_{12} .
$$

The ecological interpretation of the parameter inequalities in Theorem 5(b) and (c) is that interspecific competition is sufficiently strong (as measured by $c_{12}$ and $c_{21}$ ), relative to intraspecific competition (measured by $c_{11}$ and $c_{22}$ ). This theorem suggests the Lotka/Volterra or Leslie/Gower "saddle" case of competitive exclusion (Case C in Fig. 1). However, it is possible that all the inequalities in Theorem 5 hold and there also exists 
(a)
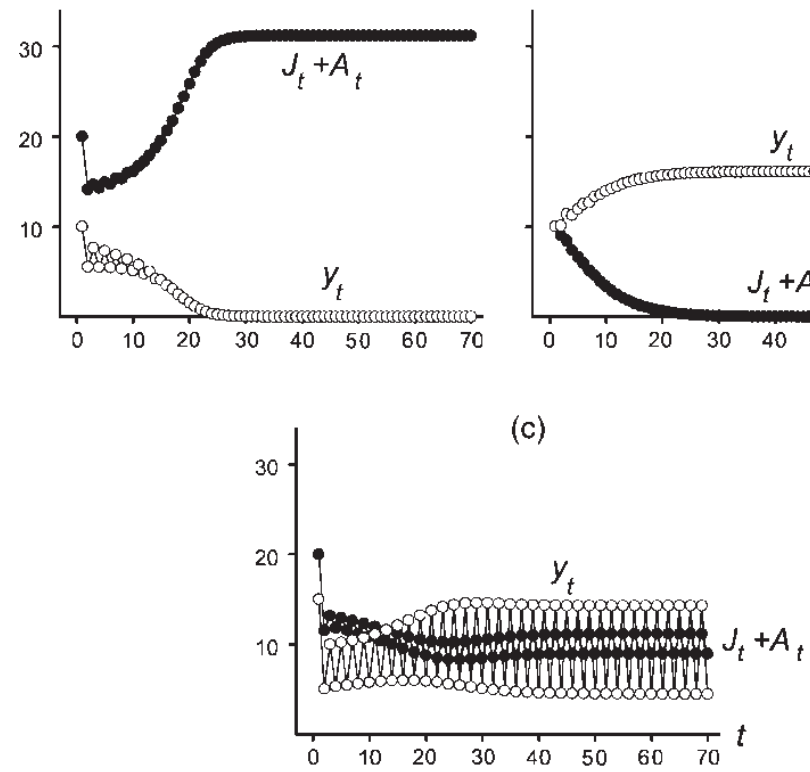

(b)

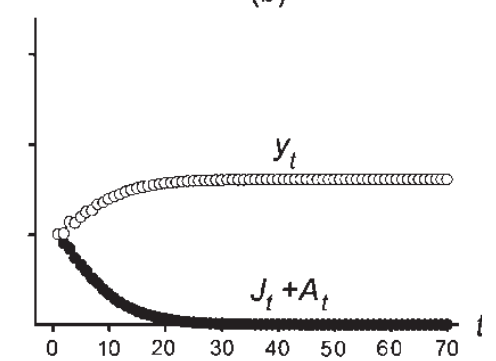

(c)

FIGURE 2 Three different initial conditions lead to solutions of the juvenile/adult Ricker competition model (7) that approach three different attractors. (a) The initial condition $\left(J_{0}, A_{0}, y_{0}\right)=(10,10,10)$ results in an orbit that approaches an equilibrium in which $y=0$, i.e. this initial condition leads to the extinction of species $y$. (b) The initial condition $\left(J_{0}, A_{0}, y_{0}\right)=(5,5,10)$ results in an orbit that approaches an equilibrium in which $J=A=0$, i.e. this initial condition leads to the extinction of species $x$. (c) The initial condition $\left(J_{0}, A_{0}, y_{0}\right)=(10,10,15)$ results in an orbit that approaches a positive two-cycle, i.e. the initial condition leads to the (non-equilibrium) coexistence of both species. The coefficients in these examples are $b_{1}=b_{2}=5, c_{11}=c_{22}=0.1, c_{12}=0.11, c_{21}=0.12$, and $\mu=0.2$.

a coexistence attractor. Figure 2 shows an example. In that figure one initial condition approaches a coexistence two-cycle, while other initial conditions lead to the extinction equilibria $E_{1}$ and $E_{2}$.

It is possible to prove the existence of coexistence two-cycles of the type observed in Figure 2. Two-cycles are fixed points of the composite map defined by the model equations (7)

$$
\begin{aligned}
b_{1}(1-\mu) J \exp \left(-c_{11}(1-\mu) J-b_{2} c_{12} y e^{-c_{21} J-c_{22} y}\right) & =J \\
b_{1}(1-\mu) A e^{-c_{11} A-c_{12} y} & =A \\
b_{2}^{2} y e^{-c_{21} J-c_{22} y} \exp \left(-b_{1} c_{21} A e^{-c_{11} A-c_{12} y}-b_{2} c_{22} y e^{-c_{21} J-c_{22} y}\right) & =y .
\end{aligned}
$$

Although it is not shown in Fig. 2, the coexistence two-cycle in that example is synchronous, i.e. the two points of the cycle are of the form

$$
\left(0, A, y_{1}\right), \quad\left(J, 0, y_{2}\right)
$$

with $J>0, A>0$, and $y_{i}>0$. "Synchronous" means that the juvenile and adult populations never appear together at the same point in time. The two-cycle equations (8) with $J=0$ reduce to the two equations

$$
\begin{aligned}
b_{1}(1-\mu) e^{-c_{11} A-c_{12} y} & =1 \\
b_{2}^{2} e^{-c_{21} J-c_{22} y} \exp \left(-b_{1} c_{21} A e^{-c_{11} A-c_{12} y}-b_{2} c_{22} y e^{-c_{21} J-c_{22} y}\right) & =1
\end{aligned}
$$


for $A$ and $y$. Using the first equation in the second we can simplify the second equation

$$
\begin{aligned}
b_{1}(1-\mu) e^{-c_{11} A-c_{12} y} & =1 \\
b_{2}^{2} \exp \left(-c_{22} y-c_{21} \frac{1}{1-\mu} A-b_{2} c_{22} y e^{-c_{22} y}\right) & =1 .
\end{aligned}
$$

Solving the first equation for

$$
A=\frac{1}{c_{11}}\left(\ln n-c_{12} y\right)
$$

substituting this result into the second equation and simplifying, we arrive at the single equation

$$
b_{2} c_{22} y e^{-c_{22} y}=\left(\frac{c_{12} c_{21}}{(1-\mu) c_{11}}-c_{22}\right) y+\left(2 \ln b_{2}-\frac{c_{21}}{(1-\mu) c_{11}} \ln b_{1}(1-\mu)\right)
$$

for $y$. A solution $y=y_{1}>0$, together with Eq. (10), yields the first point in a two-cycle (9). (If $A>0$ then this point is not an equilibrium.) The Eq. (11) can be analyzed geometrically by investigating the graphs of both sides of the equation for intersection points $y>0$. The left hand side is a positive, one humped graph passing through $y=0$ and having $y=0$ as an asymptote. The right hand side is a straight line whose slope is positive under the assumptions in Theorem 5(b) and (c). If the $y$-intercept of the straight line is positive, then either there are two intersection points of these graphs, no intersection point at all, or a tangency case of one intersection point. As parameters change and we pass from one to the other case, a bifurcation occurs at the point of tangency. (We speculate that this is a saddle node bifurcation with another two-cycle which, because it is not a synchronous cycle, is not found by the equations above.) See Figure 3 for an illustration. Note that the coexistence twocycle is created in this example by increasing the interspecific competition coefficient $c_{21}$ !

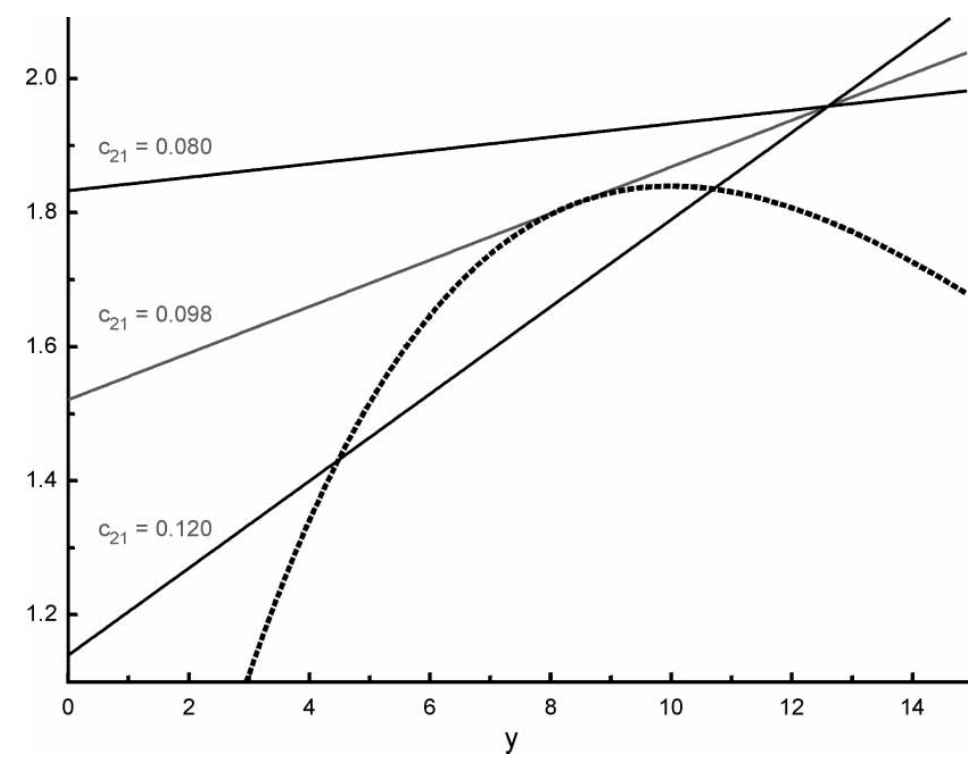

FIGURE 3 Roots $y>0$ of Eq. (9) determine synchronous two-cycles for the juvenile/adult Ricker competition model (7). The dashed line graph is that of the left hand side of this equation for the parameter values in Fig. 2. The straight line graphs are the right hand side of the equation for three selected values of the interspecific competition coefficient $c_{21}$. For the smaller value of $c_{21}$ there is no intersection and there exists no synchronous twocycle. For the larger value of $c_{21}$, two intersection points exist and give rise to a synchronous two-cycle. 
To establish the triple attractor case of interest (and observed in Fig. 2) we need to prove the stability of the two-cycle under the conditions of Theorem 5(b) and (c). The two-cycle is locally asymptotically stable if the eigenvalues of the Jacobian of the composite map are less than one in magnitude. We can calculate this Jacobian by multiplying the Jacobians of the (7) evaluated at the two points of the two-cycle. In general, this calculation is intractable, but we can carry it out for the example in Figure 2. For the parameter values in that example, Eq. (11) has the two solutions $y_{1}=4.4758$ and $y_{2}=14.304$ which produce the two-cycle points (see Eq. (9))

$$
(0,8.9396,4.4758), \quad(11.1174,0,14.304) .
$$

The Jacobians of Eq. (7) at these two points, when multiplied, yield the matrix

$$
\left(\begin{array}{ccc}
0.76624 & 0 & 0.16554 \\
0 & 0.82932 & 0 \\
-0.94822 & -1.7794 & -0.23776
\end{array}\right)
$$

whose eigenvalues $\lambda=-4.4039 \times 10^{-2}, 0.57252$, and 0.82932 are less than one in magnitude. The two-cycle is therefore locally asymptotically stable. Since the inequalities in Theorem 5(b) and (c) are satisfied by the parameters in this example the two exclusion equilibria

$$
\begin{aligned}
& E_{1}:(J, A, y)=(20.118,16.094,0) \\
& E_{2}:(J, A, y)=(0,0,16.094)
\end{aligned}
$$

are also locally asymptotically stable.

The juvenile/adult Ricker competition model shows that strong non-linearities and a time delay can produce a multiple attractor, coexistence/exclusion, scenario. According to our analysis above this scenario arises from a bifurcation that gives rise to a two-cycle coexistence state as interspecific competition is increased, which from the point of view of classical competition theory is unexpected. This same bifurcation scenario occurs in the LPA model [14]. Thus, we have two examples of competition models that provide dynamics which contradict classical competition theory in significant ways. An interesting open mathematical problem is to determine the types of competition models that possess these kinds of multiple attractors.

\section{References}

[1] H. Caswell, Matrix Population Models: Construction, Analysis and Interpretation, Second edition, Sinauer Associates, Inc. Publishers, Sunderland, Massachusetts, 2001.

[2] P. Chesson, Coexistence of competitors in spatially and temporally varying environments: a look at the combined effects of different sorts of variability, Theor. Popul. Biol., 28 (1985), 263-287.

[3] D. Clark and M. R. S. Kulenović, A coupled system of rational difference equations, Comput. Math. Appl., 43 (2002), 849-867.

[4] D. Clark, M. R. S. Kulenović and J. F. Selgrade, Global asymptotic behavior of a two-dimensional difference equation modelling competition, Nonlinear Anal., 52 (2003), 1765-1776.

[5] K. M. Crowe, A Discrete Size-Structured Competition Model, Ph.D. dissertation, Interdisciplinary Program in Applied Mathematics, University of Arizona, Tucson, 1991.

[6] J. M. Cushing, Two species competition in a periodic environment, J. Math. Biol., 10 (1980), 385-400.

[7] J. M. Cushing, Periodic Lotka-Volterra systems and time sharing of ecological niches, Lecture Notes in Biomathematics, 52 Springer, Berlin, 1983, pp. 342-348.

[8] J. M. Cushing, Periodic Lotka-Volterra competition equations, J. Math. Biol., 24 (1986), 381-403. 
[9] J. M. Cushing, A strong ergodic theorem for some non-linear matrix models for the dynamics of structured populations, Nat. Res. Modeling, 3 (1989), 331-357.

[10] J. M. Cushing, An Introduction to Structured Population Dynamics CBMS-NSF Regional Conference Series in Applied Mathematics, Vol. 71, SIAM, Philadelphia, 1998.

[11] J. M. Cushing, The LPA model, Fields Institute Commun., (To appear).

[12] J. M. Cushing, R. F. Costantino, B. Dennis, R. A. Desharnais and S. M. Henson, Chaos in Ecology Theoretical Ecology Series, Academic Press/Elsevier, San Diego, 2003.

[13] J. M. Cushing, M. Saleem, A competition model with age-structure, Lecture Notes in Biomathematics, 54 Springer, Berlin, 1984, pp. 178-192.

[14] J. Edmunds, J. M. Cushing, R. F. Costantino, S. M. Henson, B. Dennis and R. A. Desharnais, Park's Tribolium competition experiments: a non-equilibrium species coexistence hypothesis, J. Anim. Ecol., 72 (2003), $703-712$.

[15] S. N. Elaydi, An Introduction to Difference Equations, Second Edition, Springer, New York, 1999.

[16] John E. Franke and Abdul-Aziz Yakubu, Mutual exclusion versus coexistence for discrete competitive systems, J. Math. Biol., 30(2) (1991), 161-168.

[17] John E. Franke and Abdul-Aziz Yakubu, Global attractors in competitive systems, Nonlinear Anal., 16(2) (1991), 111-129.

[18] John E. Franke and Abdul-Aziz Yakubu, Geometry of exclusion principles in discrete systems, J. Math. Anal. Appl., 168(2) (1992), 385-400.

[19] John E. Franke and Abdul-Aziz Yakubu, Exclusionary population dynamics in size-structured, discrete competitive systems, J. Diff. Eqns Appl., 5(3) (1999), 235-249.

[20] H. I. Freedman, J. H. So and P. Waltman, Coexistence in a model of competition in the chemostat incorporating discrete delays, SIAM J. Math. Anal., 49 (1989), 859-870.

[21] J. Guckenheimer and P. Holmes, Nonlinear Oscillations, Dynamical Systems and Bifurcations of Vector Fields, Springer-Verlag, Berlin, 1983.

[22] J. Huisman and F. J. Weissing, Biodiversity of plankton by species oscillations and chaos, Nature, 402 (1999), 407-410.

[23] J. Huisman and F. J. Weissing, Fundamental unpredictability in multispecies competition, Am. Nat., 157 (2001), $488-494$.

[24] A. L. Koch, Competitive coexistence of two predators utilizing the same prey under constant environmental conditions, J. Theor. Biol., 44 (1974), 378-386.

[25] M. R. S. Kulenović and M. Nurkanović, Asymptotic behavior of a two dimensional linear fractional system of difference equations, Radovi Mathematićki, 11 (2002), 1-19.

[26] P. H. Leslie and J. C. Gower, The properties of a stochastic model for two competing species, Biometrika, 45 (1958), 316-330.

[27] P. H. Leslie, T. Park and D. B. Mertz, The effect of varying the initial numbers on the outcome of competition between two Tribolium species, J. Anim. Ecol., 37 (1968), 9-23.

[28] R. Levins, Coexistence in a variable environment, Am. Nat., 114 (1979), 765-783.

[29] B. Li, Periodic coexistence in the chemostat with three species competing for three essential resources, Math. Biosci., 174 (2001), 27-40.

[30] Pingzhou Liu and Saber N. Elaydi, Discrete competitive and cooperative models of Lotka-Volterra type J. Comp. Anal. Appl., 3(1) (2001), 53-73.

[31] M. Loreau and W. Ebenhoh, Competitive exclusion and coexistence of species with complex life cycles, Theor. Popul. Biol., 46 (1994), 58-77.

[32] R. McGehee and R. Armstrong, Some mathematical problems concerning the ecological principle of competitive exclusion, J. Diff. Eqns, 23 (1977), 30-52.

[33] P. de Mottoni and A. Schiaffino, Competition systems with periodic coefficients: a geometric approach, J. Math. Biol., 11 (1981), 319-355.

[34] T. Namba, Competitive coexistence in a seasonally fluctuating environment, J. Theor. Biol., 111 (1984), 369-386.

[35] T. Namba, Bifurcation phenomena appearing in the Lotka-Volterra competition equations: a numerical study, Math. Biosci., 81 (1986), 191-212.

[36] T. Namba and S. Takahashi, Competitive coexistence in a seasonally fluctuating environment. II. Multiple stable states and invasion success, Theor. Popul. Biol., 44 (1993), 374-402.

[37] T. Park, Experimental studies of interspecies competition. I. Competition between populations of the flour beetles Tribolium confusum Duval and Tribolium castaneum Herbst, Ecol. Monogr., 18 (1948), 265-308.

[38] T. Park, Experimental studies of interspecies competition. II. Temperature, humidity and competition in two species of Tribolium, Physiol. Zool., 27 (1954), 177-238.

[39] T. Park, Experimental studies of interspecies competition. III. Relation of initial species proportion to the competitive outcome in populations of Tribolium, Physiol. Zool., 30 (1957), 22-40.

[40] T. Park, P. H. Leslie and D. B. Mertz, Genetic strains and competition in populations of Tribolium, Physiol. Zool., 37 (1964), 97-162.

[41] H. L. Smith and P. Waltman, The Theory of the Chemostat: Dynamics of Microbial Competition Cambridge Studies in Mathematical Biology 13, Cambridge University Press, Cambridge, 1995.

[42] P. Waltman, Competition Models in Population Biology, CBMS-NSF regional Conference Series in Applied Mathematics, SIAM, Philadelphia, 1983. 